\title{
Final Report: Imaging of Buried Nanoscale Optically Active Materials
}

\author{
Ian Appelbaum
}

\section{Motivation:}

Semiconductor heterostructure-based light-emitting diodes (LEDs) and solid-state lasers have benefited enormously from recent developments in materials science and nanotechnology. However, methods to characterize the carrier transport through (and the light emission from) these devices on the nanoscale at which they were designed are limited by the fact that the carrier recombination occurs in layers buried deep below the semiconductor surface. Hence, processes occurring in these layers are hidden from direct observation. The development and introduction of a successful technique to examine the carrier transport and photon emission in deeply buried optically-active layers will provide a means for materials science to unmask the detailed consequences of experimentally controllable growth parameters, such as quantum dot size statistics and orientation, and defect density and charge recombination pathways.

\section{Goal:}

The project goal was to demonstrate and use Ballistic Electron Emission Luminescence microscopy to image the deeply-buried luminescent layer of semiconductor light-emitting devices with local hot electron injection from a Scanning Tunneling Microscope (STM) probe. Briefly, hot electrons are ballistically injected over a rectifying Schottky barrier into a n-i-p LED under bias. Electroluminescence from the optically active region directly below the injection point results from radiative recombination of these injected carriers. Microscopy is possible by raster-scanning the tip position.

\section{Progress:}

Integrated Photonic Detector Because the STM tunneling current is only several nA, the ballistically-injected hot-electron current is small (1-100 pA). Therefore, the luminescence intensity is weak, and external collection efficiency is reduced by the large index of refraction mismatch between semiconductors and air. To most effectively use Ballistic Electron Emission Luminescence microscopy, we must efficiently collect as much emitted luminescence as possible with an integrated photodetector. ${ }^{1}$

We have used a unique fabrication method to intimately incorporate semiconductor photodetectors with the electroluminescent hot-electron collector $p$-n structure to overcome the optical index mismatch problem in a materials-independent way. A MBE-grown AlGaAs/GaAs quantum well p-n structure obtained from IntelliEpi and a Si p-n photodetector were bonded using ultra-high-vacuum metal-film wafer-bonding. Using only several $\mathrm{nm}$ of $\mathrm{NiFe}$ to maintain optical transparency, we bond these full 2" wafers using our custom wafer manipulator, shown below in various stages of operation ex situ.

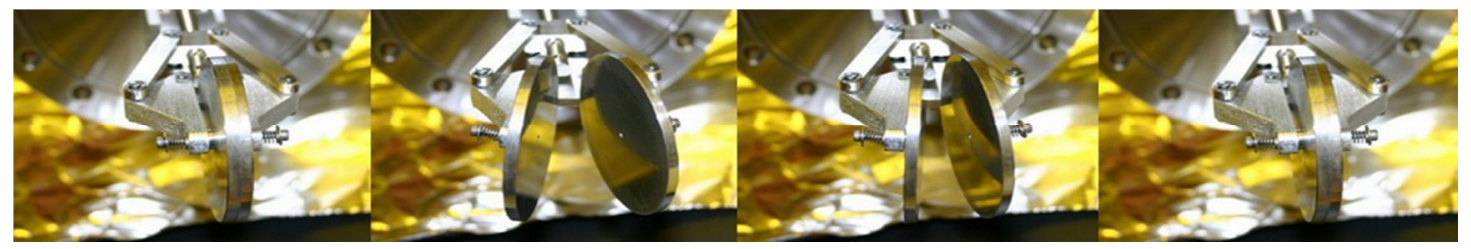

\footnotetext{
${ }^{1}$ Ian Appelbaum et al., "Vertically Integrated Optics for Ballistic Electron Emission Luminescence Microscopy",
} Appl. Phys. Lett 86, 063110 (2005) 
After this bonding, the handle wafer can be etch away to expose the n-type surface for forming hot-electron pass-filter Schottky barriers. To verify proper injection and luminescence mechanisms before scanning microscopy is attempted, we first fabricate tunnel-junction devices which use $\mathrm{Al} / \mathrm{Al}_{2} \mathrm{O}_{3} / \mathrm{Al}$ evaporated (and shadow-mask patterned) structures. These devices are made from mesas defined by diamond-sawing through the bonded interface and result in arrays shown below, schematically in side-view (left) and in a micrograph in plan-view (center).
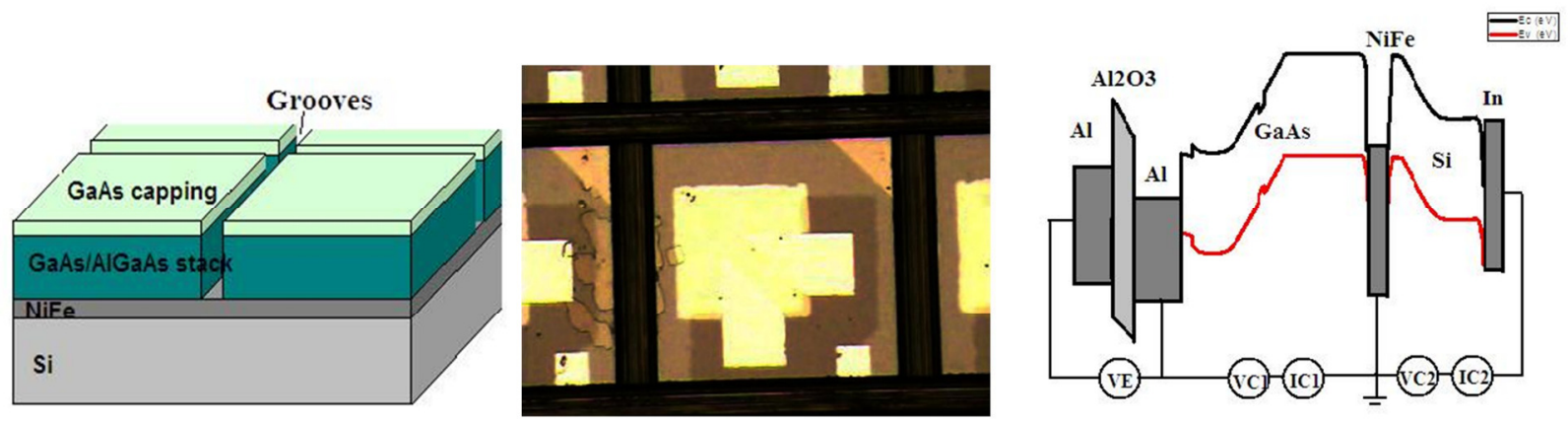

The resulting device band-diagram is also shown (right). The tunnel junction injects hot electrons from the emitter cathode on the left, once the emitter bias VE exceeds the injector Schottky barrier height. Under LED bias VC1, holes from the $\mathrm{p}+$ region are given enough potential energy to recombine with injected electrons and emit bandgap luminescence at the GaAs QW. These photons are then absorbed in the Si p-n junction on the right, constituting our BEEL signal, photocurrent IC2.
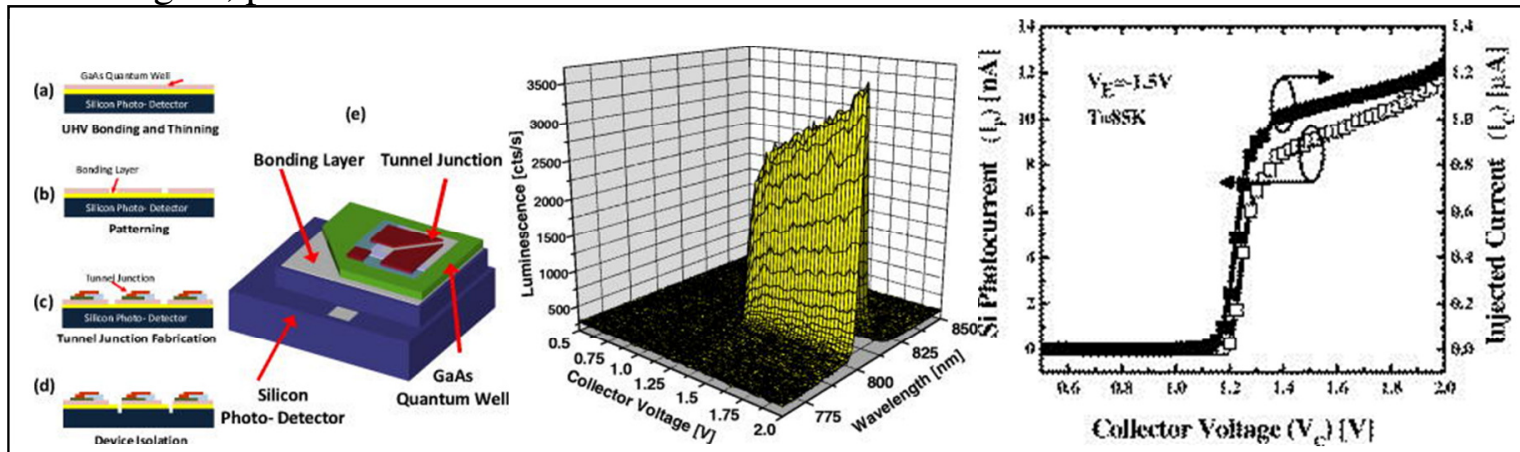

FIGURE: LEFT: (a)-(d) Device fabrication process. (e) The device scheme with a hot-electron injector on the top and a silicon $p-n$ junction photodiode at the bottom. Hot electrons injected from a tunnel junction into a QW structure induce photon emission, which are then collected by the silicon photodiode, consequently generating photocurrent signal. CENTER: Far-field luminescence measurement by an optical spectrometer with emitter bias $V_{e} \mathrm{~V}=-1.5$ at $85 \mathrm{~K}$ for different collector voltage bias on the light emitting structure. No light is generated until the collector voltage exceeds a threshold point corresponding to energy conservation. The emitted light wavelength from the GaAs QW is approximately $815 \mathrm{~nm}$. RIGHT: Collector current and photocurrent measurement at $85 \mathrm{~K}$ with $V_{e} \mathrm{~V}=-1.5$ at different collector voltage. The current thresholds are identical to the luminescence measurement, demonstrating that the heterointegrated photodetector can be used for efficient BEEL signal detection. 
Instrument: Upon transferring the grant to UMD, Ellen Williams (who has since left to become the chief scientist for BP) was added as a co-PI with the intention to maintain a relatively seamless start on the project by using the existing variable-temperature STM facilities under her control. However, after nearly a year's worth of effort, we finally concluded that her system (a modified JEOL SEM/STM/AFM) could only operate stably at tunnel currents far below those required for our local hot-electron injection experiment $(<100 \mathrm{pA}$, whereas we need $>\operatorname{lnA}$ to obtain measurable photocurrent)

In an effort to complete this project, I had to build a new microscope. Along with $\sim \$ 100 \mathrm{~K}$ of my UMD startup funds, a postdoc and student assembled an exchange-gas variable temperature STM based on a RHK head. Benchmarked operation as STM and BEEM is shown in the figure below. Despite this progress, however, all this equipment development set the scientific project back severely.

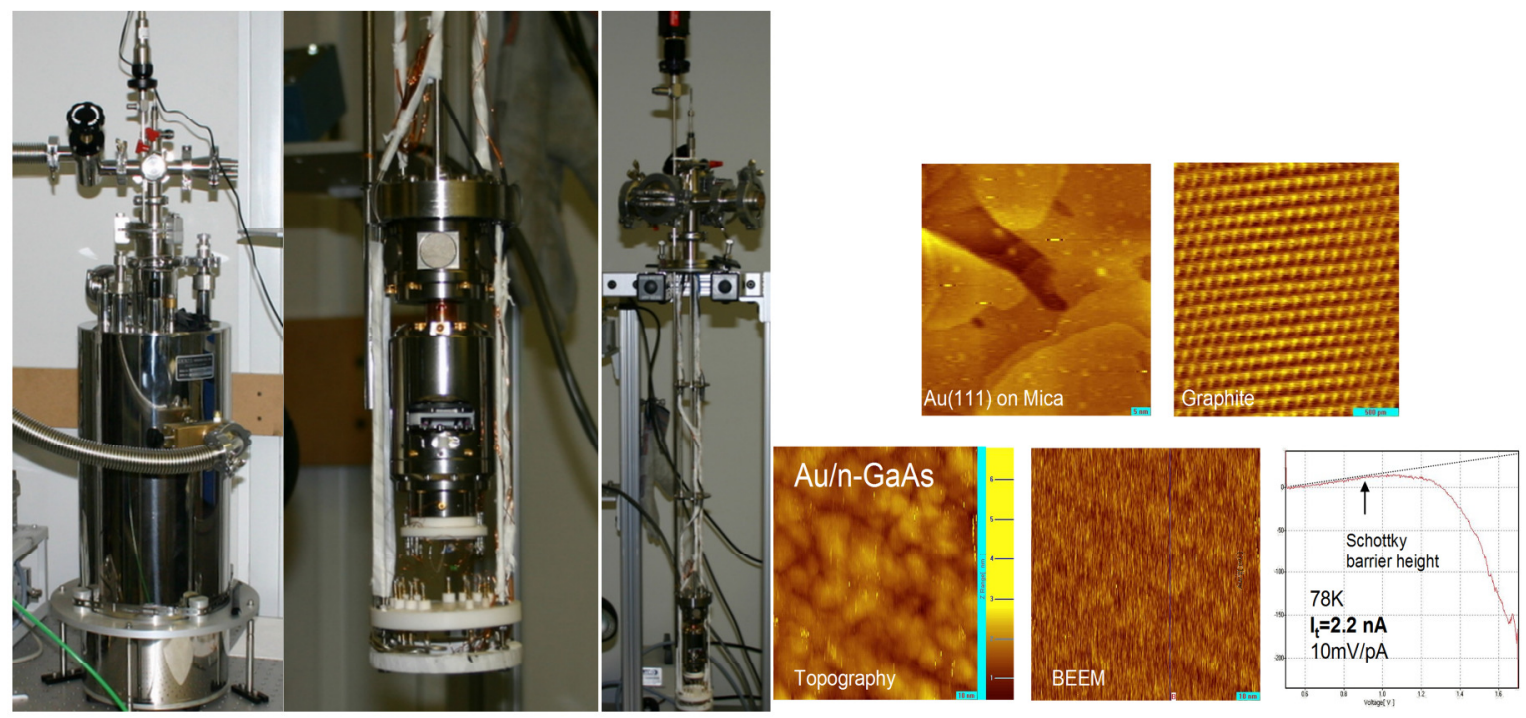

FIGURE: LEFT: Photographs of our exchange-gas variable-temperature STM/BEEM/BEEL system. RIGHT: High-resolution STM on Au/mica and graphite (showing atomic resolution at 80K), and simultaneous STM and BEEM/BEES on the canonical Au/n-GaAs system, all acquired with our custom, self-assembled system. 
Device measurement results:

By replacing the solid-state $\mathrm{Al} / \mathrm{Al} 2 \mathrm{O} 3 / \mathrm{Al}$ tunnel junction with a $\mathrm{Au}$ thin film, we use the tunneling STM tip as a source of hot electrons. However, to characterize the device operation before the time-consuming task of setting up the STM, we alternatively generate injection using internal photoemission (IPE) caused by sub-bandgap near-infrared photon absorption in the metal. At collector voltages beyond flat-band conditions, the rise in injected current corresponds to an induced photocurrent, indicating radiative recombination. These measurements are performed in-situ using a borescope to transmit light down to the mounted sample.
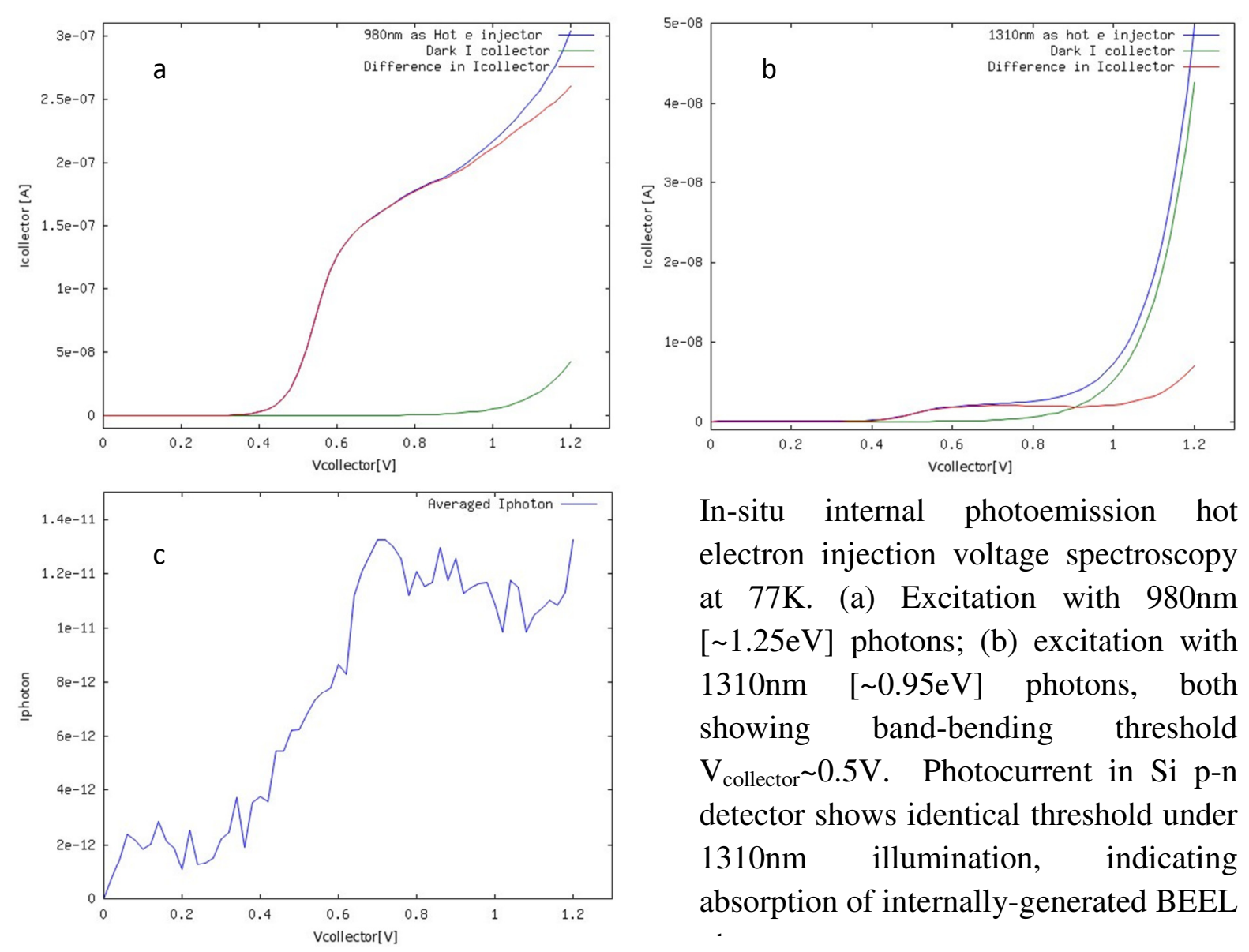

In-situ internal photoemission hot electron injection voltage spectroscopy at $77 \mathrm{~K}$. (a) Excitation with $980 \mathrm{~nm}$ $[\sim 1.25 \mathrm{eV}]$ photons; (b) excitation with $1310 \mathrm{~nm} \quad[\sim 0.95 \mathrm{eV}]$ photons, both showing band-bending threshold $\mathrm{V}_{\text {collector }} \sim 0.5 \mathrm{~V}$. Photocurrent in Si p-n detector shows identical threshold under $1310 \mathrm{~nm}$ illumination, indicating absorption of internally-generated BEEL

When the IPE source is replaced by the tunneling tip, a similar threshold in voltage spectroscopy is seen. 

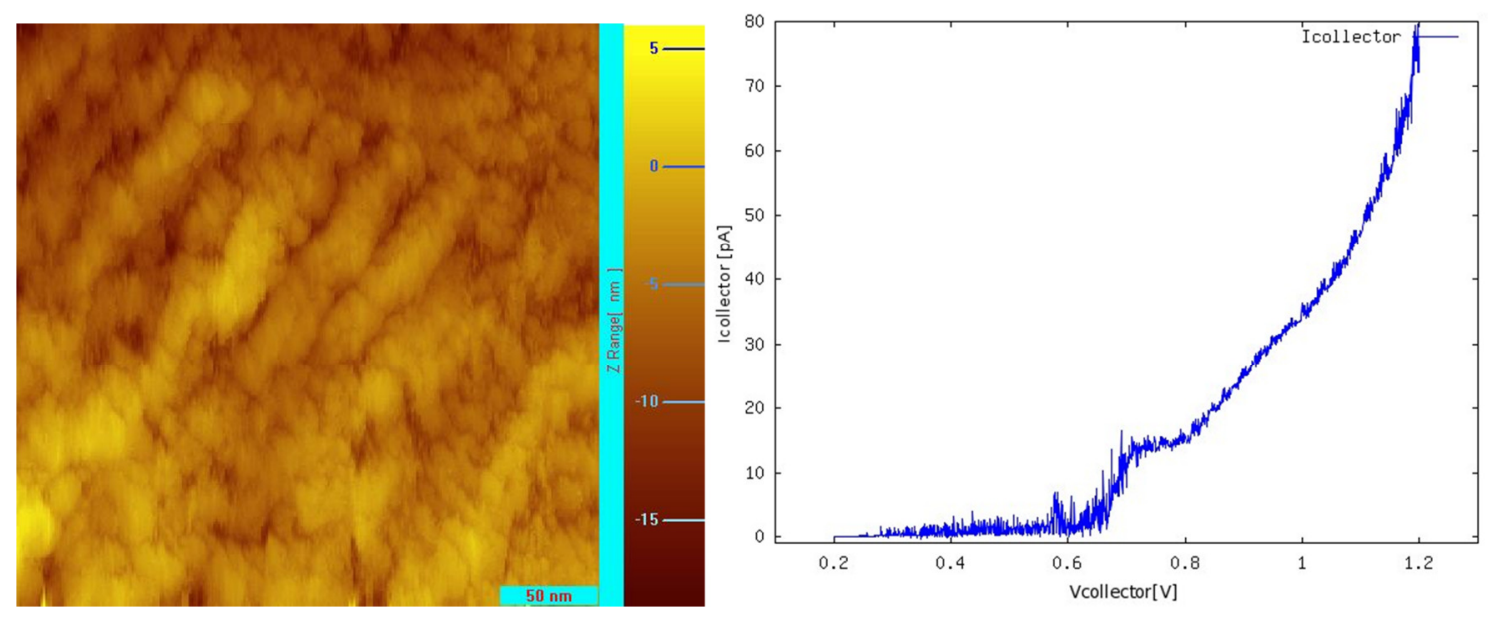

FIGURE: STM topography of BEEL device showing Au metal film morphology (LEFT) and ballistically injected current with thresholded response at slightly elevated $\sim 0.6 \mathrm{~V}$ (RIGHT). $I_{T}=$ $4.0 n A, V_{\text {tip }}=-1.4 \mathrm{~V}, 25$ average measurements, Temperature $=77 \mathrm{~K}$ 


\section{Personnel:}

This project funded portions of postdoc support (_ and one graduate
student ( Research Lab (HRL) in late 2009. took a research scientist position in Harbin University in mid-late 2010. Unfortunately, after spending the last 2 years learning how to perform the experiment and building our variable-temperature STM), Mr. suffered several personal problems and permanently left my group in 2011. Unfortunately, he is now deceased.

\section{Reference to publications of DOE-sponsored research:}

B.Q. Huang and Ian Appelbaum, "Heterointegrated near-field photodetector for ballistic electron emission luminescence”, J. Appl. Phys. 105, 086105 (2009). 


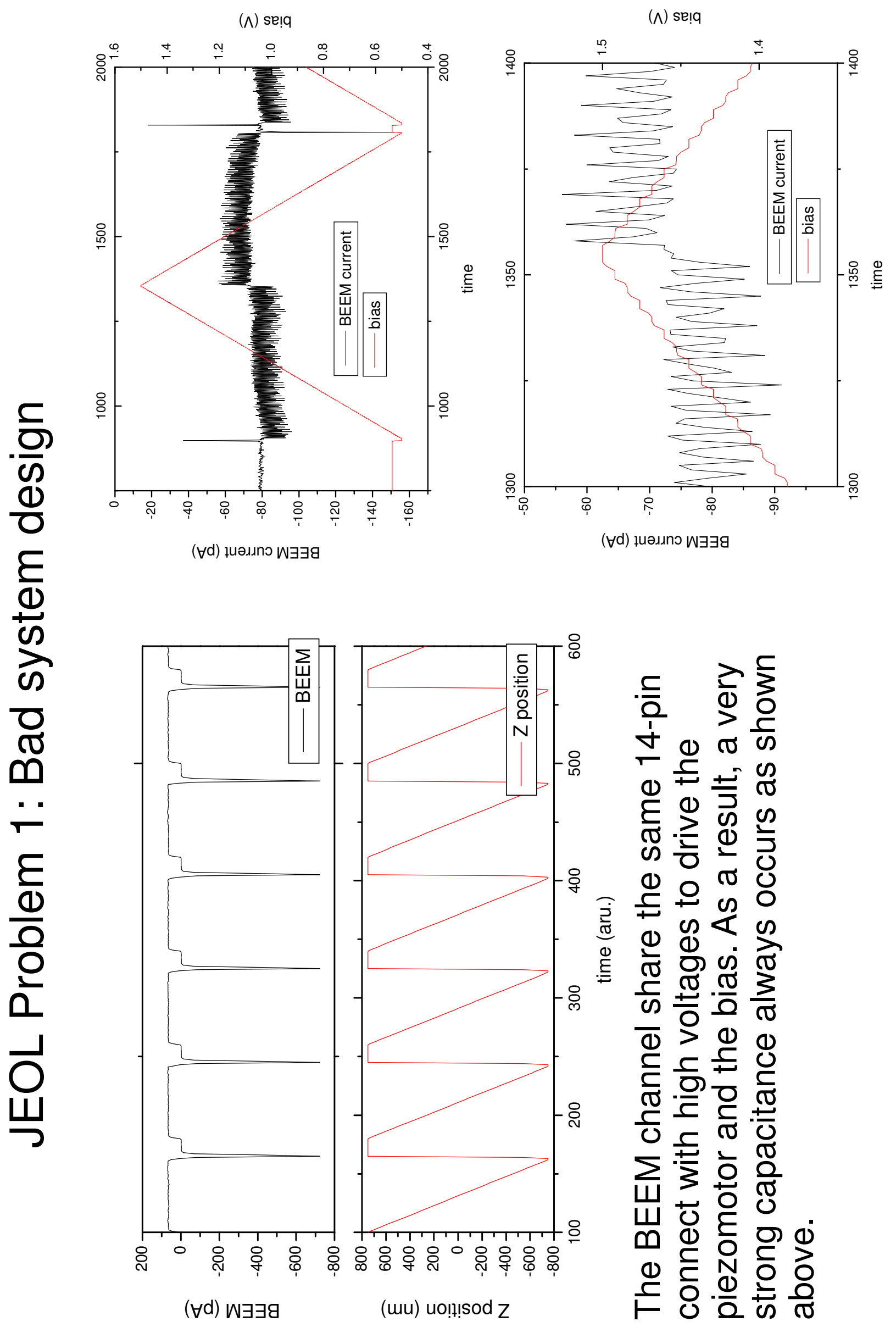




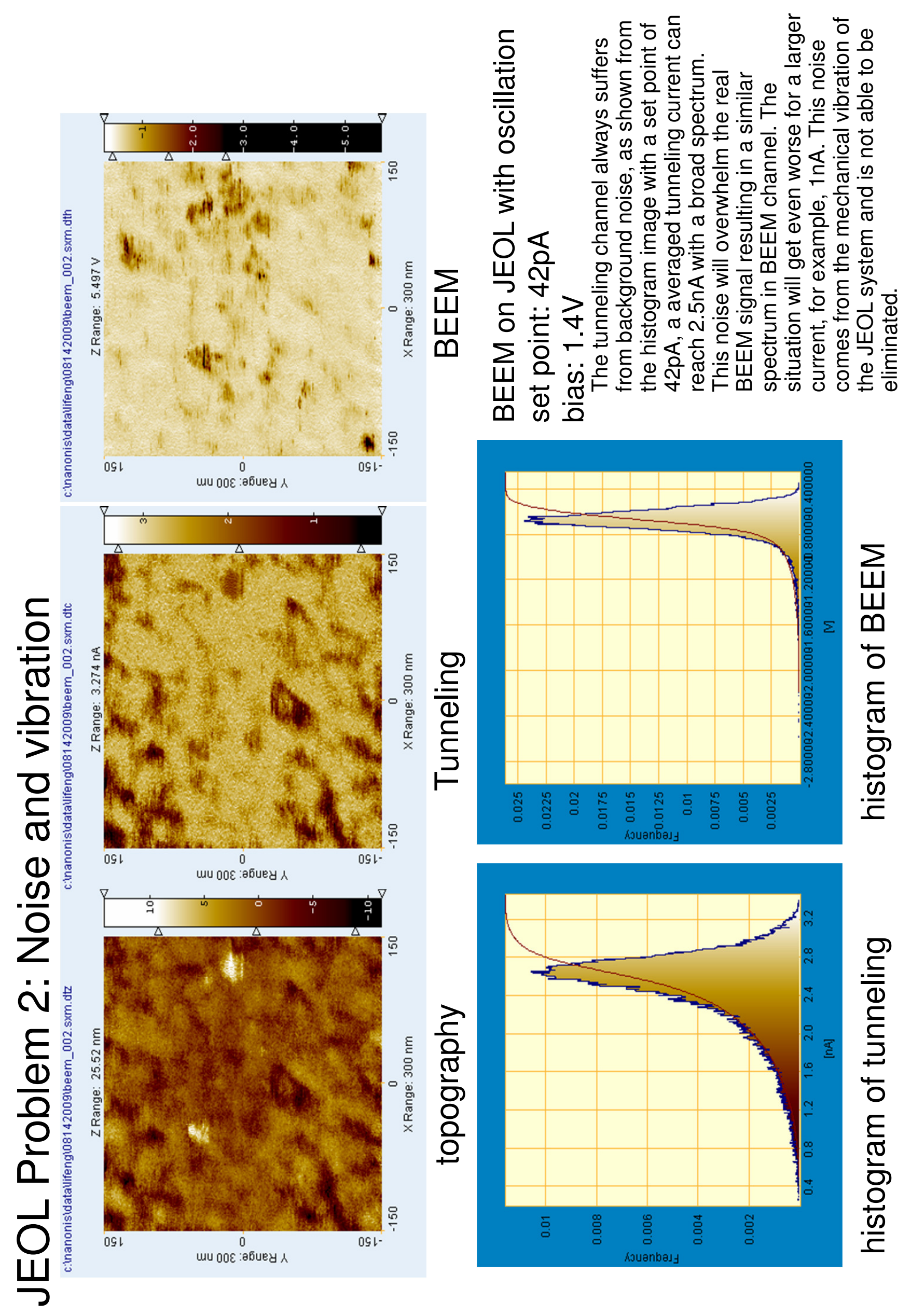



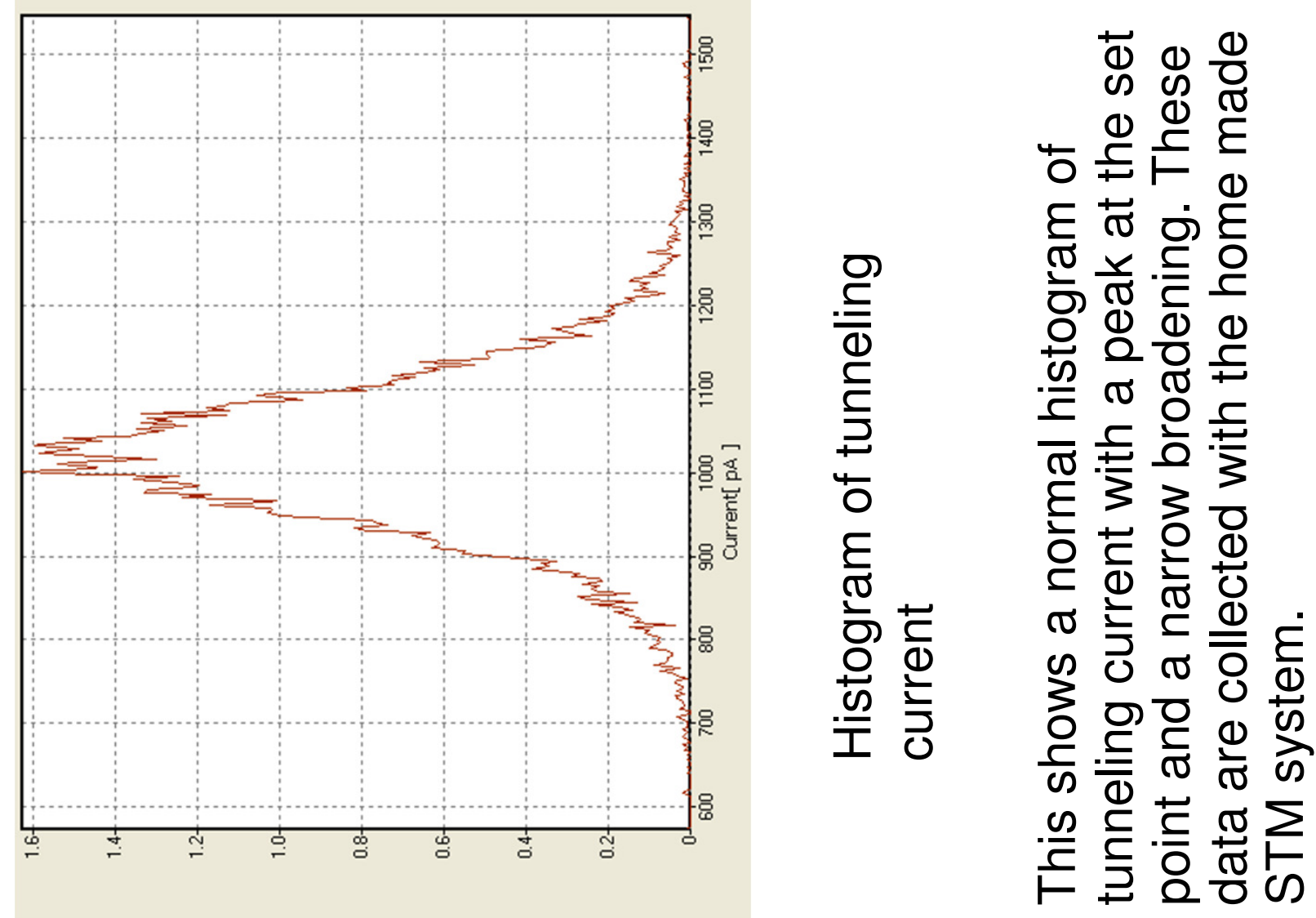

[ \% ] KJuanbaxy |ax!d
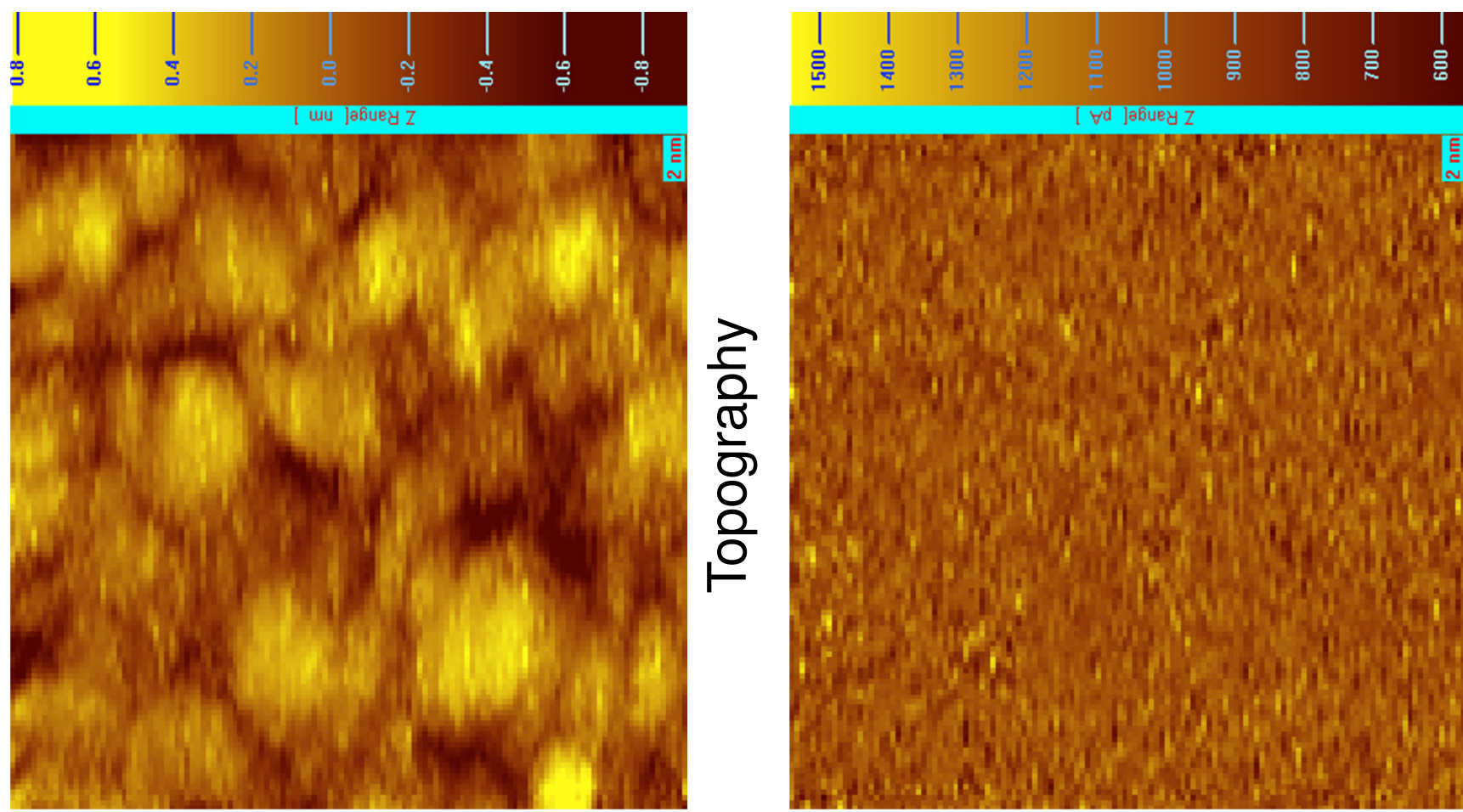